\title{
Chewing lice (Insecta, Phthiraptera) parasitizing birds in Botucatu, SP, Brazil
}

\section{Malófagos (Insecta, Phthiraptera) parasitando aves em Botucatu, SP, Brasil}

\author{
Guilherme A. Marietto-Gonçalves, ${ }^{*}$ Thiago F. Martins, ${ }^{* *}$ Raphael L. Andreatti Filho*
}

\begin{abstract}
Three hundred and ten birds were examined for chewing lice. Eleven individuals belonging to eleven species of exotic (1), and wildlife Brazilian birds (10) were positive for these ectoparasites. Ten species of chewing lice were identified (three belonging to the family Menoponidae and seven to Philopteridae). Three of these species were recognized as contaminators, and two were identified in Brazil for the first time. Distribution of these parasites and their hosts are discussed.
\end{abstract}

Keywords: ectoparasites, birds, entomology, animal health.

\section{Resumo}

Trezentas e dez aves de espécies distintas foram analisadas quanto à presença de piolhos, sendo encontradas onze parasitadas. Destas, uma espécie (1) era exótica e dez (10) eram silvestres da avifauna brasileira (10). Foram identificadas neste estudo dez espécies de piolhos (três pertencentes à família Menoponidae e sete a família Philopteridae). Destes piolhos, três espécies foram classificadas como contaminantes e duas espécies estão sendo relatadas no Brasil pela primeira vez. A distribuição destes parasitos e os seus hospedeiros habituais são discutidos neste artigo.

Palavras-chave: ectoparasitos, aves, entomologia, saúde animal.

\section{Introduction}

Chewing lice of the order Phthiraptera are obligate host-specific parasitic insects commonly observed in birds. The entire life cycle of chewing lice (i.e., egg, three nymph, and adult stages) is developed on a single host (Serra-Freire and Mello, 2006). Chewing lice usually cause severe bite-induced pruritus leading to such a discomfort that the bird can stop feeding in an attempt to free itself of the parasites (Guimarães et al., 2001; SerraFreire and Mello, 2006). It is common to observe self-mutilation of the bird that might increase the risk of secondary bacterial infection (Linardi, 2001). There are almost 4,000 species of chewing lice parasitizing birds (Price et al., 2003) and at least forty of them are of medical and economic importance for domestic birds (Price \& Graham, 1997). Two (i.e., Amblycera and Ischnocera) of the four suborders, composing the order Phthiraptera, are found on birds. Regarding the suborder Amblycera, only three (i.e., Menoponidae, Laemobothriidae and Ricinidae) of six families has species parasites from birds, while the suborder Ischnocera (which has two recognized families) only species belonging to the family Philopteridae parasitizes birds (Johnson and Clayton, 2003). There are only few reports on the geographic distributions of chewing lice worldwide, particularly in the Neotropics. Thus, the aim of this article is to describe the occurrence of chewing lice in ten species of wildlife birds belonging to native avian fauna, and one species of exotic bird in Brazil.

\section{Material and methods}

Three hundred and ten birds belonging to 67 species and 14 orders were attempted in the veterinary hospital from several origins (private owner, zoo collections and traffic seizure) and examined in the Avian Pathology Laboratory of the School of Veterinary Medicine and Animal Science (FMVZ-UNESP), São Paulo State University, Botucatu, SP, Brazil. Two hundred and two specimens belonged to wildlife native avian fauna and 88 animals were exotic birds introduced in Brazil for ornamental purposes. Parasites were collected from dead and live birds. Collection in dead birds was performed using a forceps. A pyrethroid powder (Bolfo ${ }^{\circledR}$, Bayer Healthy Care, Germain) was applied in live birds. After five minutes, the white paper sheets placed on the bottom of the cages were removed and chewing lice collected using forceps. No paper sheets were reused in order to avoid iatrogenic straggling. Specimens were fixed and conserved in $70 \%$ ethanol for further analysis. The samples is analyzed in Ixodides Laboratory of the Departament of Entomology, Instituto Oswaldo Cruz (FIOCRUZ), Manguinhos, RJ, Brazil, and the identification of chewing lice was performed based on the nomenclature by Price et al. (2003).

\footnotetext{
* Departamento de Clínica Veterinária, Faculdade de Medicina Veterinária e Zootecnia, Univ. Estadual Paulista - UNESP, Botucatu, Brazil.

** Departamento de Medicina Preventiva e Saúde Animal, Faculdade de Medicina Veterinária e Zootecnia, Universidade de São Paulo, São Paulo, Brazil. Corresponding author: Guilherme Augusto Marietto Gonçalves. Laboratório de Ornitopatologia. Departamento de Clínica Veterinária, FMVZ-UNESP, Botucatu, São Paulo, Brazil, Mailbox 560, ZIP Code: 18618-000. E-mail gmarietto_ornito@fmvz.unesp.br
} 


\section{Results and discussion}

The order, family, common name, and number of examined birds are summarized in table 1. Parasites were observed in only 11 individuals belonging to 11 different species. All but three specimens (Pavo cristatus, Ramphastos toco, and Amazona aestiva) were wildlife birds (see table 1). Results regarding suborder, family, species, number and sex of adult specimens, and number of nymphs of chewing lice are summarized in Table 2.
Occurrence of chewing lice on captive wildlife and exotic birds in Brazil has been documented (Valente et al., 2001; Brum and Rickes, 2003; Brum et al., 2003; Silva et al., 2004; Valim et al., 2005). However, these data were from bird species different than those evaluated in our work. We observed Struthiolipeurus struthionis on Rhea americana. In general, $R$. americana is the natural host for Struthiolipeurus nandu and two more Struthiolipeurus species (Price et al., 2003), but no for $S$.

Table 1: Occurrence of chewing lice on exotic and wild birds from Botucatu city during the years of 2005 to 2007 examined in the Avian Pathology Laboratory of FMVZ-UNESP

\begin{tabular}{|c|c|c|c|c|}
\hline Species & Common name & $\begin{array}{l}\text { Examined } \\
\text { birds }\end{array}$ & $\begin{array}{l}\text { Parasited } \\
\text { birds }\end{array}$ & Chewing lice genera \\
\hline \multicolumn{5}{|l|}{ Anseriformes } \\
\hline \multicolumn{5}{|l|}{ Anatidae } \\
\hline Aix spansa ${ }^{1}$ & Wood duck & 1 & - & \\
\hline Anas platyrinchus ${ }^{1}$ & Domestic duck & 5 & - & \\
\hline Anser anser ${ }^{1}$ & Greylag goose & 2 & - & \\
\hline Cairina moschata & Muscovy duck & 6 & - & \\
\hline Dendrocygna viduata & White-faced tree-duck & 1 & - & \\
\hline \multicolumn{5}{|l|}{ Charadriiformes } \\
\hline \multicolumn{5}{|l|}{ Charaiidrae } \\
\hline Vanellus chillensis & Southern lapwing & 1 & - & \\
\hline \multicolumn{5}{|l|}{ Ciconiiformes } \\
\hline \multicolumn{5}{|l|}{ Accipitridae } \\
\hline Buteo magnirostris & Roadside hawk & 2 & - & \\
\hline \multicolumn{5}{|l|}{ Ardeidae } \\
\hline Ardea cocoi & White-necked heron & 1 & - & \\
\hline Bubulcus ibis & Cattle egret & 1 & 1 & $\begin{array}{l}\text { Ciconiphilus decimfasciatus } \\
\text { (Boisduval and Lacordaire, 1835) }\end{array}$ \\
\hline Butorides striatus & Striated heron & 1 & - & \\
\hline Egretta thula & Snowy egret & 1 & 1 & Ciconiphilus decimfasciatus \\
\hline Syrigma sibilatrix & Whistling heron & 1 & - & \\
\hline Tigrisoma fasciatum & Rufescent tiger-heron & 1 & - & \\
\hline \multicolumn{5}{|l|}{ Falconidae } \\
\hline Falco sparverius & American kestrel & 1 & - & \\
\hline Polyborus plancus & Crested caracara & 1 & - & \\
\hline \multicolumn{5}{|l|}{ Columbiformes } \\
\hline \multicolumn{5}{|l|}{ Columbidae } \\
\hline Columba livia ${ }^{1}$ & Rock pigeon & 7 & - & \\
\hline Columbina talpacoti ${ }^{1}$ & Ruddy ground-dove & 1 & - & \\
\hline Streptopelia risoria ${ }^{1}$ & Ringneck dove & 1 & - & \\
\hline \multicolumn{5}{|l|}{ Craciformes } \\
\hline Cracidae & & & & \\
\hline
\end{tabular}




\begin{tabular}{l|l|c|c|l}
\hline Species & Common name & $\begin{array}{c}\text { Examined } \\
\text { birds }\end{array}$ & $\begin{array}{c}\text { Parasited } \\
\text { birds }\end{array}$ & Chewing lice genera \\
\hline Penelope superciliaris & Rusty-marginated guan & 1 & 1 & $\begin{array}{l}\text { Menopon gallinae }{ }^{2} \\
\text { (Linnaeus, 1758) }\end{array}$ \\
& & & & Menacanthus spp. \\
& & & Goniodes spp. ${ }^{2}$
\end{tabular}

\section{Cuculiformes}

Cuculidae

Guira guira

Guira cuckoo

1

Squirrel cuckoo

1

Piaya cayana

\section{Galliformes}

Phasianidae

Pavo cristatus $^{1}$

Indian peafowl

2

Guinea fowl

Numida meleagridis ${ }^{1}$

\section{Gruiformes}

Cariamidae

Cariama cristata

Red-legged seriema

1

$1 \quad$ Heptapsogaster frielingi

(Eichler, 1941);

Tinamotaecola wardi

(Hellenthal, Price and Timm, 2002)

Rallidae

Aramides cajanea

Gallinula melanops

Porphyrio martinica

Gray-necked wood rail

Spot-flanked gallinule

Purple gallinule

2

1

1

\section{Passeriformes}

Cardinalidae

Saltator similis

Green-winged saltator

6

Corvidae

Cyanocorax cristatellus

Emberezidae

Coryphospingus cucullatus

Sicalis flaveola

Sporophila angolensis ${ }^{1}$

Sporophila caerulescens

Sporophila lineola

Sporophila maximiliani ${ }^{1}$

Sporophila nigricollis

Volatinia jacarina

Zonotrichia capensis

Curl-crested jail

Red-crested finch

Saffron finch

Lesser seed-finch

Double-collared seedeater

Lined seedeater

Great-billed seed-finch

Yellow-bellied seedeater

Blue-black grassquit

Rofous-collared sparrow
Osborniella guiraensis

(Kellogg, 1906)

Amyrsidea spp. 


\begin{tabular}{|c|c|c|c|c|}
\hline Species & Common name & \begin{tabular}{|c|} 
Examined \\
birds
\end{tabular} & $\begin{array}{l}\text { Parasited } \\
\text { birds }\end{array}$ & Chewing lice genera \\
\hline \multicolumn{5}{|l|}{ Estrildidae } \\
\hline Lonchura striata $^{1}$ & White-rumped munia & 2 & - & \\
\hline Erythrura gouldiae ${ }^{1}$ & Goudian finch & 30 & - & \\
\hline Taeniopygia guttata $^{1}$ & Zebra finch & 3 & - & \\
\hline \multicolumn{5}{|l|}{ Fringilidae } \\
\hline Carduelis carduelis ${ }^{1}$ & European goldfinch & 1 & - & \\
\hline Carduellis magellanica & Hooded siskin & 40 & - & \\
\hline Serinus canaria ${ }^{1}$ & Canary & 20 & - & \\
\hline \multicolumn{5}{|l|}{ Icteridae } \\
\hline Molothrus bonariensis & Shiny cowbird & 1 & - & \\
\hline Gnorimopsar chopi & Chopi black-bird & 1 & - & \\
\hline \multicolumn{5}{|l|}{ Muscicapidae } \\
\hline Turdus leucomellas & Pale-breasted thrush & 1 & - & \\
\hline Turdus rufiventris & Rufous-bellied thrush & 2 & - & \\
\hline \multicolumn{5}{|l|}{ Thraupidae } \\
\hline Euphonia violacea & Violaceus euphonia & 1 & - & \\
\hline \multicolumn{5}{|l|}{ Piciformes } \\
\hline \multicolumn{5}{|l|}{ Picidae } \\
\hline Colaptes campestris & Campo flicker & 1 & - & \\
\hline \multicolumn{5}{|l|}{ Ramphastidae } \\
\hline Ramphostos toco ${ }^{b}$ & Toco toucan & 9 & 1 & $\begin{array}{l}\text { Columbicola columbae } \\
\text { (Linnaeus, 1758) }\end{array}$ \\
\hline \multicolumn{5}{|l|}{ Psittaciformes } \\
\hline \multicolumn{5}{|l|}{ Cacatuidae } \\
\hline Nynphicus hollandicus ${ }^{1}$ & Cockatiel & 5 & - & \\
\hline \multicolumn{5}{|l|}{ Psittacidae } \\
\hline Agapornis fisheri ${ }^{1}$ & Fisher's lovebird & 2 & - & \\
\hline Agapornis personata ${ }^{1}$ & Blacked-masked lovebird & 2 & - & \\
\hline Agapornis roseicollis $^{1}$ & Peach-faced lovebird & 1 & - & \\
\hline Amazona aestiva $^{1}$ & Blue-fronted amazon parrot & 12 & 1 & $\begin{array}{l}\text { Paragoniocotes semicingulatus } \\
\text { (Piaget, 1890) }\end{array}$ \\
\hline Amazona amazonica $^{1}$ & Orange-winged amazon parrot & 2 & - & \\
\hline Amazona xanthops ${ }^{1}$ & Yellow-faced amazon parrot & 1 & - & \\
\hline Aratinga leucophtalmus & White-eyed conure & 8 & - & \\
\hline Brotogeris tirica & Plain parakeet & 1 & - & \\
\hline Melopsittacus undulatus ${ }^{1}$ & Budgerigar & 1 & - & \\
\hline Neophema elegans ${ }^{1}$ & Elegant parrot & 1 & - & \\
\hline Psittacula krameri ${ }^{1}$ & Ring-neck & 1 & - & \\
\hline \multicolumn{5}{|l|}{ Strigiformes } \\
\hline \multicolumn{5}{|l|}{ Strigidae } \\
\hline Athene cunicularia & Burrowing owl & 1 & 1 & $\begin{array}{l}\text { Strigiphilus desertae } \\
\text { (Carriker, 1966) }\end{array}$ \\
\hline
\end{tabular}




\begin{tabular}{|c|c|c|c|c|}
\hline Species & Common name & $\begin{array}{l}\text { Examined } \\
\text { birds }\end{array}$ & $\begin{array}{l}\text { Parasited } \\
\text { birds }\end{array}$ & Chewing lice genera \\
\hline \multicolumn{5}{|l|}{ Tytonidae } \\
\hline Tyto alba & Barn owl & 2 & 1 & $\begin{array}{l}\text { Strigiphilus aitkeni } \\
\text { (Clay, 1966) }\end{array}$ \\
\hline \multicolumn{5}{|l|}{ Struthioniformes } \\
\hline \multicolumn{5}{|l|}{ Rheidae } \\
\hline Rhea americana & Greater rhea & 4 & 1 & $\begin{array}{l}\text { Struthiolipeurus nandu } \\
\text { (Eichler, 1950) }\end{array}$ \\
\hline \multicolumn{5}{|l|}{ Trochiliformes } \\
\hline \multicolumn{5}{|l|}{ Trochilidae } \\
\hline Eupetomena macroura & Swallow-tailed hummingbird & 6 & - & \\
\hline
\end{tabular}

${ }^{1}$ captive birds, 2parasite species considered as contamination.

Table 2: Chewing lice species observed on exotic and wild birds from Botucatu city during the years of 2005 to 2007 examined in the Avian Pathology Laboratory of FMVZ-UNESP

\begin{tabular}{lllccc}
\hline \hline & \multicolumn{2}{c}{ Phthiraptera } & \multicolumn{3}{c}{ Samples } \\
\hline Subordem & Family & Species & 0 & + & Nymphs \\
Amblycera & Menoponidae & Amyrsidea spp. & - & 1 & 4 \\
& & Ciconiphilus decimfasciatus & 1 & 4 & - \\
& & Menacanthus spp. & - & 2 & 1 \\
& & Menopon gallinae & 4 & 2 & - \\
Ischnocera & Osborniella guiraensis & 7 & 6 & 8 \\
& \multirow{2}{*}{ Philopteridae } & Columbicola columbae & 7 & 6 & 1 \\
& & Goniodes spp. & - & - & 2 \\
& & Heptapsogaster frielingi & 4 & 3 & 2 \\
& & Paragoniocotes semicingulatus & 6 & 15 & - \\
& & Strigiphilus aitkeni & 5 & 6 & 3 \\
& Strigiphilus desertae & 7 & 7 & 9 \\
& Tinamotaecola wardi & 1 & 2 & 2 \\
\hline \hline & Struthiolipeurus nandu & 1 & 2 & - \\
\hline
\end{tabular}

struthionis, whose main host is the ostrich (Dominguez-De-Tena et al., 1976; Mey, 1998). Parasitism by $S$. struthionis on $R$. americana was previously described in Brazil (Sinkoc et al., 2005), but this phenomenon was considered as straggling.

Ciconiphilus decimfasciatus can be found on 32 species of egrets e.g., Bubulcus ibis and Egretta thula (Price and Beer 1965; Price et al., 2003). This chewing louse was recently found by Albano et al. (2005) on Great egret (Ardea alba), on the State of Rio Grande do Sul, Brazil.

Both chewing lice, Heptapsogaster frielingi and Tinamotaecola wardi (Hellenthal et al., 2002) found on Cariama cristata in our study were previously reported in specimens from Brazil.

Both Strigiphilus aitkeni found on Tyto alba, and Paragoniocotes semicingulatus found on Amazona aestiva were already reported in Brazil (Guimarães, 1947; Clay, 1966). On the other hand, neither $S$. desertae found on Athene Some chewing lice were found on unexpected hosts (Price et al., 2003). This phenomenon was defined by Hopkins (1939) and Pilgrim and Palma (1982) as "straggling" and "contamination", respectively. The term "straggling" is used when chewing lice are found on an atypical host. The "stragglers" are the result of an infestation in captive birds not related with human handling. It mainly occurs when birds of different species are placed together. The term "contamination" is used when chewing lice infest uncommon hosts due to human handling. Cases of "straggling" i.e., Menopon gallinae, and Goniodes spp. cunicularia nor Osborniella guiraensis collected on Guira guira were previously mentioned in Brazil, and so are reported in this study for the very first time.

Absence of male specimens of Amyrsidea spp. impeded specific identification of chewing lice collected from Pavo cristatus. Although P. cristatus is often infested by two Amyrsidea species (Scharf and Price, 1983, Price et al., 2003), it was not possible to determine to which species the chewing lice specimens belong. 
parasitizing Penelope superciliaris; and Columbicola columbae parasitizing Ramphastos toco, are shown in Chart 1.

There is no report of chewing lice on $P$. superciliaris. Thus, we believe that the presence of $M$. gallinae and Goniodes sp. on this host is not natural and represents a case of straggling since they are found exclusively on Galliformes, family Phasianidae (Clay, 1940; Emerson, 1954).

Although Valim et al. (2005) had reported Menacanthus chaparensis on $P$. superciliaris, the specimens of Menacanthus spp. were morphologically more similar to those found on the order Galliformes, family Phasianidae, than to those typically found on Craciformes.

Columbicola columbae on $R$. toco is a typical case of straggling. Austrophilopterus is the only genus of the family Philopteridae found on the family Ramphastidae, order Piciformes (Carriker, 1950; Price and Weckstein, 2005), and the genus Columbicola is exclusively found on birds of the family Columbidae, order Columbiformes (Tendeiro, 1955; Clayton and Price, 1999).

We infer that straggling cases in our study are easily justified due to voluntary or involuntary exposure of wildlife birds to urban and/or farm environments. So, we found chewing lice from nonnative species parasitizing wildlife native species. Identification of parasitic fauna is critical due to the ecologic importance of these biological entities in the control of populations of wildlife birds, as well as to identify exotic species parasitizing native birds. Our study contributes to the epidemiological mapping of avian chewing lice and represents the first description in the region of Botucatu, São Paulo state, Brazil.

\section{Acknowledgements}

We thanks the collaboration of Michel P. Valim from MZUSP, for the identification of the lice species presented herein.

\section{References}

ALBANO, A.P.N.; BRUM, J.G.W.; COIMBRA, M.A.A. First report of Ciconiphilus decimfasciatus (Amblycera: Menoponidae) in Casmerodius albus (Aves: Ciconiiformes) from Brazil. Arquivos do Instituto Biológico, v. 72, p. 263-264, 2005.

BRUM, J.G.W.; RICKES, E.M. Laemobothrion glutinans Nitzsch, 1861 e Cuculiphilus alternatus (Osborn, 1902) (Mallophaga: Amblycera) em Urubu (Coragyps atratus) em área do Parque Zoológico do Rio Grande do Sul. Arquivos do Instituto Biológico, v. 70, p. 497-498, 2003.

BRUM, J.G.W.; VALENTE, A.L.; PAULSEN, R.M.M.; MULLER, G. Malófagos parasitos de alguns animais silvestres no estado do Rio Grande do Sul. Arquivos do Instituto Biológico, v. 70, p. 177-178, 2003.

CARRIKER JÚNIOR, M.A. Studies in Neotropical Mallophaga. No. VI. suborder "Ischnocera". Family "Philopteridae". Arquivos do Instituto Biológico, v.10, p.163-188, 1950.

CLAY, T. Genera and species of Mallophaga occurring on Gallinaceous hosts - Part II. Goniodes. Proceedings of the Zoological Society of London (Series B), v.110, p.1-120, 1940.

CLAY, T. The species of Strigiphilus (Mallophaga: Philopteridae) parasitic on the barn owls Tyto (Tytonidae). Journal of the Entomological Society of Queensland, v. 5, p. 10-17, 1966.

CLAYTON, D.H.; PRICE, R.D. Taxonomy of New World Columbicola (Phthiraptera: Philopteridae) from the Columbiformes (Aves), with descriptions of five new species. Annals of the Entomological Society of America, v. 92, p. 675-685, 1999.

DOMINGUEZ-DE-TENA, M.; HERNANDEZ-RODRIGUEZ, S.; BECERRA-MARTELL, C.; CALERO-CARRETERO, R.; MORENOMONTANEZ, T.; MARTINEZ-GOMEZM, F. Struthiolipeurus nandu Eichler, 1950 (Mallophaga; Philopteridae) parasitising an ostrich (Struthio camelus) in the Cordoba zoo, Spain. Revista Iberica de Parasitologia, v. 36, p. 167-173, 1976.

EICHLER, W. Zur Klassifikation der Lauskerfe (Phthiraptera Haeckel: Rhynchophthirina, Mallophaga und Anoplura). Archiv für Naturgeschichte, v.10, p. 345-398, 1941.

EMERSON, K.C. A review of the genus Menopon Nitzsch, 1818 (Mallophaga). Annals and Magazine of Natural History (Series 12), v. 7, p. $225-232,1954$
GUIMARÃES, L.R. Contribuições ao conhecimento dos Malófagos dos psitácidas brasileiros. I - Espécies da família Philopteridae (Ischnocera). Arquivos de Zoologia, v. 5, p. 243-309, 1947.

GUIMARÃES, J.H.; TUCCI, E.C.; BARROS-BATTESTI, D.M. Ectoparasitos de Importância Veterinária. São Paulo: Editora Plêiade, 2001.

HELLENTHAL, R.A.; PRICE, R.D.; TIMM, R.M. Review of the chewing louse genus Tinamotaecola (Phthiraptera:Philopteridae) with the description of the three new species. Journal of the Kansas Entomological Society, v. 74, p. 136-141, 2002.

HOPKINS, G.H.E. Straggling in the Mallophaga. Entomologist, v. 72, p. 75-77, 1939.

JOHNSON, K.P.; CLAYTON, D.H. The biology, ecology, and evolution of chewing lice. In: PRICE, R.D.; HELLENTHAL, R.A.; PALMA, R.L.; JOHNSON, K.P.; CLAYTON, D.H. The chewing lice: world checklist and biological overview. Illinois: Illinois Natural History Survey Special Publication, 2003. p. 449-476.

LINARDI, P.M. Piolhos (Sugadores e Mastigadores). In: MARCONDES, C.B. Entomologia Médica e Veterinária. São Paulo: Atheneu, 2001. p. 183-238.

MEY, E. Zur taxonomie, Lebensweise und parasitophyletischen evidenz der federlingsgattung Struthiolipeurus sensu lato (Insecta, Phthiraptera, Ischnocera). Mitteilungen aus dem Museum für Naturkunde in Berlin, Zoologische Reihe, v. 74, p. 65-93, 1998.

PILGRIM, R.L.C.; PALMA, R.L. A list of the chewing lice (Insecta: Mallophaga) from birds in New Zealand. National Museum of New Zealand Miscellaneous Series 6, p.1-32, 1982.

PRICE, M.A.; GRAHAM, O.H. Chewing and sucking lice as parasites of mammals and birds. USDA Agricultural Research Service, Technical Bulletin, v. 1849, p. 1-257, 1997.

PRICE, R.D.; BEER, J.R. A review of Ciconiphilus (Mallophaga: Menoponidae). Canadian Entomologist, v. 97, p. 657-666, 1965.

PRICE, R.D.; WECKSTEIN, J.D. The genus Austrophilopterus Ewing (Phthiraptera: Philopteridae) from toucans, toucanets, and araçaris (Piciformes: Ramphastidae). Zootaxa, v. 918, p. 1-18, 2005.

PRICE, R.D.; HELLENTHAL, R.A.; PALMA, R.L. World checklist of chewing lice with host associations and keys to families and genera. The chewing lice: world checklist and biological overview. In: PRICE, R.D.; HELLENTHAL, R.A.; PALMA, R.L.; JOHNSON, 
K.P.; CLAYTON, D.H. The chewing lice: world checklist and biological overview. Illinois: Illinois Natural History Survey Special Publication, 2003. p. 1-448.

SCHARF, W.C.; PRICE, R.D. Review of the Amyrsidea in the subgenus Argimenopon (Mallophaga: Menoponidae). Annals of the Entomological Society of America, v. 76, p. 441-451, 1983.

SERRA-FREIRE, N.M.; MELLO, R.P. Entomologia \& Acarologia. Rio de Janeiro: L.F. Livros, 2006.

SILVA, S.O.; OLIVEIRA, H.H.; TEIXEIRA, R.H.F.; SERRA-FREIRE, N.M. Malófagos parasitas de aves campestres cativas do Zoológico Municipal Quinzinho de Barros, Sorocaba, Estado de São Paulo, Brasil. Entomology and Vectors, v. 11, p. 333-339, 2004.

SINKOC, A.L.; MULLER, G.; BRUM, J.G.W.; SOARES, M.P.; OLIVEIRA, L.T.; GONÇALVES, I.P.D. Ocorrência de Struthiolipeurus rheae (Phthiraptera: Ischnocera) em Rhea americana no Rio Grande do Sul. Arquivos do Instituto Biológico, v. 72, p. 497-498, 2005.
TENDEIRO, J. Estudos sobre malófagos. Revisão monográfica do género Columbicola Ewing (Ischnocera, Philopteridae). Memórias da Junta de Investigações do Ultramar, v. 32, p. 461-462, 1965.

VALENTE, A.L.S.; PAULSEN, R.M.M.; BRUM, J.G.W. Colpocephalum maculatum Piaget, 1880 (Mallophaga: Menoponidae) em Gavião-carijó (Rupornis magnirostris) e Caracará (Polyborus plancus) no Rio Grande do Sul. Arquivos da Faculdade de Veterinária da UFRGS, v. 29, p. 147-148, 2001.

VALIM, M.P.; TEIXEIRA, R.F.H.; AMORIM, M.; SERRA-FREIRE, N.M. Malófagos (Phthiraptera) recolhidos de aves silvestres no Zoológico de São Paulo, SP, Brasil. Revista Brasileira de Entomologia, v. 49, p. 584-587, 2005. 